\title{
Programa educativo de estimulación prenatal para el desarrollo cognitivo y sensorial en Potosí
}

\author{
Educational program of prenatal stimulation for cognitive and sensory development \\ in Potosí
}

\author{
Programa educacional de estimulação pré-natal para o desenvolvimento cognitivo e \\ sensorial em Potosí
}

Gelitza Isabel Huaylla Fuertes
huayllafuertesgelitza@gmail.com
https://orcid.org/0000-0001-9525-553X

Universidad Privada Domingo Savio, Potosi, Bolivia
Recibido: octubre 2020 Revisado: noviembre 2020 Aceptado: diciembre 2020 Publicado: enero 2021
RESUMEN

La estimulación prenatal promueve el desarrollo sensorial e intelectual del bebé mientras esté en el útero materno, lo cual incidirá en toda su vida. A pesar de la relevancia hay desconocimiento entre las mujeres gestantes y el personal de salud. El servicio de salud de Potosí no desarrolla programas educativos en esta área. Por ello, el objetivo la presente investigación fue proponer un programa educativo de estimulación prenatal para el fortalecimiento del desarrollo cognitivo y sensorial del nuevo ser en mujeres gestantes atendidas en los centros de salud del Servicio Departamental de Salud de Potosí. Para ello se planteó una investigación descriptiva desde un enfoque mixto (CuantitativoCualitativo). Una vez analizados los datos se procedió a diseñar el programa de formación, el cual se estructuró en tres fases. Se concluye que el Servicio Departamental de Salud no oferta servicios de Estimulación prenatal.

Palabras clave: Educación; Estimulación prenatal; mujeres gestantes
Prenatal stimulation promotes the baby's sensory and intellectual development while in the womb, which will affect the baby's entire life. Despite its relevance, there is a lack of knowledge among pregnant women and health professionals. The health service in Potosí does not develop educational programs in this area. Therefore, the objective of this research is to propose an educational program of prenatal stimulation to strengthen the cognitive and sensory development of the new being in pregnant women attended at the health centers of the Departmental Health Service of Potosí. For this, a descriptive research was proposed from a mixed approach (Quantitative-Qualitative). After analyzing the data, the training program was designed, structured in three phases. It is concluded that the Departmental Health Service does not offer prenatal stimulation services.

Key words:

Education; Prenatal stimulation; pregnant women

\section{RESUMO}

A estimulação pré-natal promove o desenvolvimento sensorial e intelectual do bebê ainda no útero, - que afetará toda a sua vida. Apesar de sua relevância, existe desconhecimento entre gestantes e profissionais de saúde. O serviço de saúde de Potosí não desenvolve programas educacionais nesta área. Portanto, o objetivo desta pesquisa é propor um programa educativo de estimulação pré-natal para fortalecer o desenvolvimento cognitivo e sensorial do novo ser em gestantes atendidas nos centros de saúde do Serviço de Saúde Departamental de Potosí. Para isso, foi proposta uma pesquisa descritiva a partir de uma abordagem mista (Quantitativa-Qualitativa).. Uma vez analisados os dados, foi elaborado o programa de treinamento, que foi estruturado em três fases. Conclui-se que o Serviço de Saúde Departamental não oferece serviços de estimulação pré-natal.

Palavras-chave:

Educação; Estimulação pré-natal; mulheres grávidas 


\section{INTRODUCCIÓN}

Dentro de la Psicopedagogía uno de los aspectos fundamentales es generar un aprendizaje infantil que contribuya al desarrollo óptimo de las diversas áreas, especialmente el área cognitiva y sensorial; por ser una de las más importantes. El desarrollo del ser humano comienza en el momento de la concepción y la etapa prenatal es de suma importancia por la cantidad y rapidez de los cambios que se producen. Los nueve meses de gestación son clave, ya que un alto porcentaje de las alteraciones futuras en el aprendizaje tienen su origen en este periodo. En tal sentido, la estimulación prenatal resulta indispensable para un mejor aprendizaje; por ello es necesario que la futura madre estimule al bebé desde la etapa intrauterina dadas las repercusiones que conlleva este proceso.

La principal característica que define la estimulación prenatal es que se realiza durante el embarazo una atención temprana que está pensada para mejorar o prevenir los probables déficits en el desarrollo sensorial y mental de niños con riesgo de padecerlos, consiste en crear un ambiente estimulante, para que su desarrollo se vea potenciado desde esta etapa. Para González (2016):

Es muy importante que se realicen actividades para estimular al bebé en el segundo trimestre de embarazo, las que incluyen procesos que afectan el desarrollo visual del pequeño y otros sentidos, como el oído, donde la voz de la mamá, la música, la presión y la luz cumplen un papel fundamental. Mediante esta comunicación con el bebé, éste aprende a reconocer y responder a patrones creados por ella o por su papá, los que perfeccionan su desarrollo físico y mental (p. 47).

A pesar de la relevancia de la Estimulación Prenatal, aún existe una carencia de conocimiento entre las mujeres gestantes y el personal de salud. Si bien existen en el Servicio Departamental de Salud Potosí (SEDES) Bolivia, algunos centros de salud que tienen unidades para la atención a menores de 5 años; no se desarrollan programas educativos de estimulación prenatal para mujeres gestantes. De modo que, muchas mujeres dejan de lado el cuidado en el embarazo, frecuentemente debido a mitos y creencias. En este proceso la madre tiene un rol fundamental, desde el momento de la concepción del nuevo ser, en conjunto con los aportes del profesional preparado para potenciar el desarrollo cognitivo y sensorial.

Al realizar una consulta preliminar sobre esta temática a mujeres gestantes y profesionales destaca algunos aspectos observados; tales como: a.- La mayoría de las mujeres gestantes desconocen el término "estimulación prenatal"; lo que lleva a que no realicen dicha estimulación; b.- En el contexto sociocultural donde se encuentran las mujeres gestantes no se practica la estimulación prenatal; c.- Se desconoce la incidencia de la estimulación en el futuro de los ser por nacer; d.No se reconoce la importancia de la asistencia profesional en la estimulación prenatal; e.- Se requiere difundir entre las madres gestantes que los ejercicios y técnicas para los niños/as pueden, desde el vientre, mejorar el aprendizaje temprano de los circuitos neurológicos que se alcanzan a través de los sentidos y los movimientos, lo cual les permitirá lograr una mayor adaptación al mundo que lo rodea; f.- Actualmente muchos profesionales del área de salud también desconocen de la importancia de este tema; esto se debe a la poca preparación para poder aplicar estimulación prenatal. Es importante la capacitados para ejecutar programas en esta área, dados que contribuye a una mejor atención integral de la madre y el nuevo ser, incluso incide en un mejor pronóstico del desarrollo del niño/a.

Como se puede observar en esta aproximación a la problemática de investigación las ideas 
identificadas fueron difusas y sin explicación teórica o científica; lo cual denota la necesidad de generar programas educativos que le proporcione herramientas a las madres gestantes y a los profesionales de la salud. En tal sentido, se establecieron las siguientes interrogantes:

1. ¿Cuáles son los fundamentos teóricos del proceso de estimulación prenatal en el desarrollo cognitivo y sensorial del nuevo ser en mujeres gestantes?

2. ¿Cuál es el estado actual del desarrollo cognitivo y sensorial del nuevo ser en mujeres gestantes atendidas en los centros de salud del Servicio Departamental de Salud de Potosí?

3. ¿Qué componentes conformarán el programa educativo de estimulación prenatal para fortalecer el desarrollo cognitivo y sensorial en mujeres gestantes atendidas en los centros de salud del Servicio Departamental de Salud de Potosí?

A partir de estas interrogantes se formuló como objetivo general: Proponer un programa educativo de estimulación prenatal para el fortalecimiento del desarrollo cognitivo y sensorial del nuevo ser en mujeres gestantes atendidas en los centros de salud del Servicio Departamental de Salud de Potosí. Como objetivos específicos se plantearon los siguientes: 1. Establecer los fundamentos teóricos del proceso de estimulación prenatal en función al desarrollo cognitivo y sensorial del nuevo ser en mujeres gestantes. 2. Determinar el estado actual del desarrollo cognitivo y sensorial del nuevo ser en las mujeres gestantes atendidas en los centros de salud del Servicio Departamental de Salud de Potosí. 3. Identificar los componentes que conformarán el programa educativo de estimulación prenatal para fortalecer el desarrollo cognitivo y sensorial en mujeres gestantes atendidas en los centros de salud del Servicio Departamental de Salud de Potosí.
Se espera que la presente investigación proporcione herramientas para aumentar el bienestar de las madres gestantes y el desarrollo cognitivo y sensorial del nuevo ser; a través de un programa educativo para la estimulación prenatal que contará con una guía virtual para poder proporcionar más información sobre los beneficios de la estimulación prenatal. Desde el ámbito científico el programa educativo será un aporte al área de salud, pues el Servicio Departamental de Salud (SEDES) Potosí no tiene acciones educativas en los centros de salud dirigidas a la estimulación prenatal. Desde el ámbito social la investigación contribuirá de manera significativa al desarrollo del nuevo ser; de manera que, las mujeres gestantes tendrán una mayor oportunidad de fortalecer el desarrollo sensorial y cognitivo de sus hijos.

Los fundamentos teóricos de la investigación se centraron en las ciencias asociadas a la salud y su vinculación con la educación. En tal sentido, el primer elemento de estudio es el proceso de embarazo que según García (2019) es el periodo comprendido entre la fecundación de un óvulo y el nacimiento del recién nacido; durante 40 semanas, aproximadamente 9 meses. Por lo que el embarazo puede también ser definido como un proceso de cambios físicos, sociales y emocionales en la madre y el feto. Por lo que desde el inicio la mujer debe tener mayor cuidado en su estado físico y emocional para no afectar al bebé o nuevo ser; además debe estimular a su bebe desde antes del nacimiento porque el embarazo es una de las etapas más preciosas en la vida de una mujer. Este proceso en distinto en cada mujer; sin embargo en el caso de madres adolescentes hay una particular atención porque muchas veces son embarazos no es planificados, ni deseado y no se dan en las mejores condiciones físicas y emocionales. Para Romero (2007), pocas madres "acuden a control prenatal y su inmadurez física causa problemas de salud que no se resuelven durante el embarazo" (p. 80). Es así que lo primero a trabajar con las 
mujeres gestantes adolescentes son los aspectos emocionales en la aceptación de su bebe, para poco a poco ir estimulándolo y trabajar con ambos (madre-hijo).

Por otra parte, desde la fecundación el niño posee una su carga genética que debe ser desarrollada a su máximo potencial en todas las áreas y brindarle la mejor calidad de aprendizaje infantil temprano. La estimulación prenatal son un conjunto de acciones que proporcionara al niño las experiencias que este necesita para potenciar sus capacidades y facultades que posee en su carga genética, los órganos de los sentidos y los centros cerebrales (García, 2008). Por ello se puede decir que el bebé puede tener mejores condiciones en su desarrollo no solo a nivel físico sino psicológico y social. Sin embargo, cada gestante presenta características fisiológicas, psicológicas, culturales y sociales ligadas a creencias y prácticas propias de su contexto; esto incide en las prácticas de cuidado sobre estimulación prenatal. A pesar de los diferentes cuidados y prácticas culturales la estimulación puede aprovechar al máximo las relaciones con los bebes dentro el útero. Es así que la estimulación prenatal desarrolla el sentido del oído a través de la voz materna, sobre todo para que se puedan crear vínculos afectivos.

En la presente investigación, dicha estimulación prenatal tiene sus bases teóricas en las ideas de Piaget (1969) quien señala que todo aprendizaje se basa en experiencias previas, entonces, mediante la estimulación se le proporcionará al feto situaciones que le inviten al aprendizaje. Por otra parte, este autor también hace referencia al crecimiento neuronal que consiste en la formación de nuevos y más complejos circuitos neuronales. Igualmente se base en la Neurociencia y la Pedagogía Prenatal, la primera se ocupan del sistema nervioso, sus aspectos y funciones especializadas e involucra tanto a la biología del sistema nervioso como a las ciencias humanas, sociales y exactas para contribuir a una mejor calidad de vida durante todo el ciclo vital. La segunda área donde se sustenta la investigación señala que nuestra educación comienza antes del nacimiento, es decir, durante la etapa prenatal donde las relacionales de las capacidades intelectuales, incluso de la creatividad se desarrollan en la etapa de gestación (Bertín, 2018).

En base a estas ideas, los estudios realizados en la segunda mitad del siglo XX determinaron que el bebé siente y reacciona desde el útero y sí se estimula al feto este responderá a ciertos estímulos; lo que provoca información que llegará al cerebro del niño y permitirá memorizar ciertos patrones sencillo y cortos. Esto evidencia que la educación se inicia en la etapa intrauterina para un mejor desenvolvimiento después de nacer. De esta manera, ocurre un desarrollo sensorial que puede ser estimulado desde el tercer a cuarto mes de gestación, el cual se atribuye a trabajar los sentidos con diferentes técnicas sencillas donde se presentan diferentes beneficios después del nacimiento del niño o niña. Las técnicas de estimulación prenatal son variadas e inciden en distintos aspectos del campo sensorial; sin embargo, los estímulos deben ser sencillos y se deben presentar de forma organizada.

La estimulación auditiva (hablar con el feto o nuevo ser) permite reconocer las voces y sonidos más familiares. El feto reacciona ante estímulos luminosos (desde el cuarto mes de gestación) y táctiles, desde la sexta a décima semana de gestación puede estimularse presionando en el vientre materno con las manos, con masajes y desde el quinto mes de embarazo (ejercicios suaves, equilibrio, gimnasia de embarazadas) etc.

Todo incide positivamente en el desarrollo cognitivo, el cual hace referencia a las millones de células que adquieren función desde que la madre tiene contacto con su hijo en el vientre. Ahí se crean conexiones sinápticas a partir del desarrollo 
del sistema nervioso central; así lo revelan estudios que este se crece en el cuarto mes de gestación.

Las neurociencias se ocupan del sistema nervioso, así como de cada uno de sus aspectos y funciones especializadas. Involucran tanto a la Biología del sistema nervioso, como a las Ciencias Humanas, Sociales y Exactas que, en conjunto, representan la posibilidad de contribuir al bienestar humano por medio de mejoras en la calidad de vida durante todo el ciclo vital (UNESCO, 1995, p. 78).

Tomando en cuenta, lo señalado hasta ahora se puede decir que, el proceso de estimulación prenatal es un conjunto de acciones desarrolladas en la etapa gestacional. Donde se va estimulando al bebé con diferentes actividades dentro el útero para ir desarrollando el área cognitiva y sensorial. Estimulando los sentidos durante los nueve meses de gestación a partir del segundo a tercer mes de embarazo, a través de un conjunto de técnicas sensoriales y psicoactivas junto a estrategias de relajación que la madre, el padre, o ambos realizan para crear un vínculo amoroso con su hijo no nacido, con el objetivo que proporcionan seguridad e influir positivamente en el desarrollo cognitivo del niño/a por nacer (Guzmán Bobadilla de Mejía, 2014).

Desde los años 80 diversos investigadores vienen trabajando entorno a la percepción de los bebés; los resultados afirman que si perciben y reaccionan ante los estímulos extrauterinos. Es así como encontramos los trabajos de Verny (1988), quien trabajó la audición del bebé en el vientre y observó una reacción de distención fetal. Por otra parte, la estimulación temprana crea cambios en el cerebro, luego de estimular la audición fetal y de observar una reacción sensorial intrauterina (Corominas, 2011). Van de Carr desarrolló una metodología de estimulación prenatal luego de observar la reacción fetal ante el tacto abdominal (Gútiez, 2016). Por otro lado, los doctores Brent Logan, director del Instituto Prenatal en Seattle y Thomas Verny, fundador de la Asociación Norteamericana de Psicología Prenatal y Perinatal, se han dedicado a investigar los efectos del ambiente externo sobre el feto y la riqueza de la estimulación prenatal (Kinedu, 2017).

Al iniciar el trabajo de la estimulación prenatal se deben tomar en cuenta los factores físicos, sociales y emocionales de la madre gestante. El factor físico porque para lograr buena estimulación prenatal se debe trabajar con la madre; ya que los efectos de la estimulación en el útero quedarían anulados, si la madre descuida su alimentación. La mala alimentación de la madre repercute en el posterior desarrollo del aprendizaje de los niños y niñas y el futuro desenvolviendo cotidiano; también presentan efectos adversos a nivel del sistema neuronal al ser que los niños malnutridos (Rodríguez, 2018 y López Fuentes, 2016). El factor social está asociado a las madres cuyo embarazo fue o no planificado y en la aceptación de su bebé. Esto generalmente se da cuando una adolescente queda embarazada y debe afrontar el peso negativo de la sociedad. En el factor emocional se debe tomar en cuenta que durante nueve meses de embarazo las mujeres pasan por numerosas fases que pueden afectar al bebé, tales como: la angustia, el miedo, el estrés, la tristeza, la inseguridad; aunque también afectan la alegría, la tranquilidad y la calma. Por este motivo cada vez más expertos apuntan que sería recomendable tener en cuenta también la salud emocional de las futuras madres y no sólo su estado físico y su alimentación (López Fuentes, 2016).

El trabajo en estimulación prenatal aborda sobre todo en el desarrollo cognitivo y sensorial, así como se procesa la información a partir de la percepción y su relación con la parte sensorial y las funciones cognitivas. Por ello el Proceso 
de Estimulación se deben plantear técnicas que involucran a la madre y al bebé. Es por ello que todo trabajo de estimulación prenatal corresponde a dos líneas: Una dirigida a la madre y sus estados anímicos; y la otra al feto para el aumento las primeras conexiones neuronales que se desarrollan dentro del vientre a partir de la semana diez y siete.

Entre los beneficios que arroja esta estimulación se encuentra: Una mayor conexión entre el bebé y la madre, donde el niño sepa cuanto antes que es querido y que habita en un lugar seguro y agradable; los bebes desarrollan mayor seguridad en las áreas visual, lingüística, auditiva y motora; ciclos de sueño más regulares y descansos más profundos; una mayor capacidad de atención y por lo tanto de aprendizaje.

LaEducaciónylasalud son pilaresfundamentales que están íntimamente relacionadas; la primera es la ciencia que estudia los procesos para la transmisión de conocimiento, los hábitos, las costumbres y los valores de una sociedad a la siguiente generación. La segunda es la ciencia que investiga los procesos para el logro del bienestar biopsicosocial; por lo cual no solamente está enfocada a eliminación y/o prevención de enfermedades, sino en garantizar el derecho fundamental a la vida y el logro del grado más alto posible de salud. La relación entre estas áreas es muy importante para poder formar, información, educación y comunicar a la ciudadanía sobre su salud.

Por ello, el desarrollo infantil temprano se debe acudir a áreas como la Psicopedagogía, la cual proporciona el apoyo necesario para trabajar la Estimulación Prenatal.

Para Verny y Van de Carr (2016):

Los programas en educación prenatal facilitan un mayor desarrollo optimizando en el desarrollo de los sentidos, que son la base para el aprendizaje en las diferentes áreas (viso perceptivo, auditivo, lingüística y motora). Favorecen el desarrollo de la comunicación, la coordinación viso motriz, la memoria y el razonamiento, producen diferentes efectos en la morfología funcional del cerebro potenciando su desarrollo, habilidades y las formas de interactuar (p. 16).

Existen diferentes tipos de intervención psicopedagógica que tiene que ver con las necesidades psicológicas como las educativas para el nuevo ser. En este caso con la psicopedagogía se podrá colaborar y fortalecer en el desarrollo del niño desde una edad temprana (desarrollo prenatal) ayudándoles de muchas formas desde crear un clima favorable para la madre y el niño, como también por medio de sentimientos, emociones y sensaciones favorables.

Lamentablemente, indagando más se puede decir que en Bolivia aún no existen servicios de salud públicos relacionados específicamente al tema y al tratamiento de la estimulación prenatal. Sin embargo, en algunos departamentos como Santa Cruz, Cochabamba y La Paz atienden de cierto modo algunos programas como: "Partos y bebés sin dolor" es un concepto que motiva las sesiones de yoga, charlas y estimulación prenatal para mamás embarazadas en la Caja Nacional de Salud (CNS) de Cochabamba. En Potosí el área de salud aun no desarrolla este tipo de servicios, sin embargo, el SEDEGES Potosí con los Centros de Desarrollo brinda servicios de trabajo con bebés a partir de lo seis meses hasta los cuatro años donde también participan algunas mujeres embarazadas para tratamientos y seguimientos psicoprofilacticos. Por ello, la presente investigación se desarrolló en el Servicio Departamental de Salud Potosí (SEDES), institución rectora y gestora de la Salud en el Departamento; la cual regula e implementa políticas, planes, programas de salud, promueve la participación de todos los sectores públicos, privados y sociales 


\section{MÉTODO}

La presente investigación fue de tipo descriptivo - propositivo y fue realizada desde un enfoque mixto (Cuantitativo-cualitativo). Se asumió el enfoque mixto porque en la investigación se recabaron datos cuantitativos para conocer y medir cuantas mujeres conocen la importancia de la estimulación prenatal. Por otra parte, los datos cualitativos permitieron explorar la realidad de las mujeres gestantes atendidas en los centros de salud del municipio de Potosí y de los profesionales, para obtener información sobre su conocimiento entorno a la importancia de la estimulación prenatal. En tal sentido, se estableció como variable la estimulación prenatal; para luego aplicar las técnicas de revisión documental, encuesta y entrevista semiestructurada a través de los siguientes instrumentos: Cuestionario y Guía de entrevista. La población de la presente investigación es de tipo finita porque solo se consideró una institución, los Servicio Departamental de Salud (SEDES) Potosí. Dicha institución cuenta con un total de 8051 mujeres gestantes atendidas, 23 jefes Médicos y 23 Licenciadas en Enfermería de los diferentes. La muestra fue representativa de tipo de muestreo probabilístico y quedó conformada por 95 mujeres gestantes, 12 médicos y 20 enfermeras.

El cuestionario formó parte del diagnóstico y permitió recabar información sobre el conocimiento que manejaban los participantes en cuanto a la estimulación prenatal, la importancia y los beneficios que aportaría un programa educativo a las mujeres gestantes que son atendidas por los centros de salud del municipio de Potosí. Este instrumento fue aplicado a 95 madres gestantes y 20 enfermeras del Servicio Departamental de Salud de Potosí.

Por otra parte, la Guía de Entrevista también formó parte del diagnóstico para recabar información acerca del conocimiento que manejaban los médicos sobre la importancia y los beneficios de la estimulación prenatal. El instrumento se aplicó a médicos de los Centros de Salud que atienden a las mujeres gestantes; se tomó una muestra representativa que corresponde a 12 médicos.

\section{RESULTADOS}

Los resultados obtenidos del cuestionario nos indica que el $50 \%$ de las mujeres participantes se encuentran en 21 y 33 años, lo que indica posibles embarazos planificados; sin embargo, el $25 \%$ de las encuestadas son mujeres entre 15-20 años este es un porcentaje significativo, pues pueden ser embarazos no planificados de adolescentes. Por otra parte, más del $50 \%$ de mujeres gestantes encuestadas indican desconocimiento acerca de la estimulación prenatal; a su vez los profesionales participante aunque manifiestan tener conocimiento sobre estimulación prenatal, no manejan información más amplia del tema.

Igualmente destaca que en dos componentes sensoriales, más del $50 \%$ de las mujeres hablan a sus bebés y acarician su vientre; esto se ve ratificado por los profesionales quienes señalan que el componente auditivo es el más empleado en las mujeres. Lo que significa que la mayoría de las mujeres aplican una sola técnica en cada componente sensorial. Por lo tanto, se estaría perdiendo un gran beneficio para sus futuros niños por el desconocimiento de otras técnicas sensoriales.

En el aspecto desarrollo cognitivo se encontró que el $60 \%$ de mujeres gestantes encuestadas realizan rutinas suaves de ejercicio como ayuda para su proceso de embarazo. Por su parte, los profesionales señalan que las funciones cognitivas a estimular son la percepción (45\%) y la memoria (30\%); en un menor porcentaje indican que las funciones cognitivas a estimular son el lenguaje 
(20\%) y la atención (5\%). Las cuatro funciones cognitivas son clave el desarrollo de los bebés en el vientre materno; sin embargo, es un área que aún se desconoce en el sector salud de modo que no se informa a las mujeres gestantes sobre técnicas cognitivas para estimular a los bebés perdiendo los grandes beneficios que la estimulación aporta para los futuros aprendizajes.

En el aspecto valoración de la estimulación prenatal, el $100 \%$ de los profesionales encuestados desean poder tener un programa de estimulación prenatal en sus Centros de Salud. Consideran que es importante poder ampliar los conocimientos sobre estimulación prenatal y brindar un apoyo a las mujeres gestantes para el desarrollo de sus bebés.

Por otra parte, la guía de entrevista permitió recabar la opinión de los médicos de los diferentes centros de salud sobre la estimulación prenatal; la cual se organizó en cuatros grandes dimensiones estudiados: Acciones Técnicas; Desarrollo Sensorial, Desarrollo Cognitivo, Estimulación Prenatal. Con relación a las acciones técnicas los médicos indican que tienen conocimiento sobre la estimulación prenatal, conocen algunas técnicas que deben realizar las mujeres gestantes para obtener un mejor desarrollo de las capacidades a nivel cognitivo y sensorial de los bebes, así mismo consideran que son importantes y aportan muchos beneficios al desarrollo del nuevo ser. Pero podemos remarcar de manera puntual y clara que no hay un conocimiento real y precisa de los elementos o componentes que debe tener un programa de estimulación prenatal el cual sería de gran impacto para la sociedad con niños preparados para sus aprendizajes en un mundo mucho más competitivo.

En la dimensión de Desarrollo Sensorial los médicos afirman que los órganos sensoriales que pueden ser estimulados son el oído, visual y táctil para un mejor desarrollo del área cognitiva del lenguaje y la coordinación, lo cual es valioso para la presente investigación. Sin embargo, los conocimientos que presentan los profesionales son escasos y limitados, por lo que no brindan la información adecuada sobre las técnicas sensoriales a las mujeres gestantes para estimular a sus hijos beneficiándolos en su desarrollo. Por lo que es necesario ampliar conocimientos de estimulación prenatal para coadyuvar al desarrollo de los futuros niños.

En la dimensión desarrollo cognitivo los médicos desconocen del área cognitiva, sin embargo, indican que se pueden estimular algunas funciones como: el lenguaje y la memoria que ayudan al intelecto de los futuros niños. Es importante y necesario ampliar conocimiento sobre las técnicas en esta área, ya que son de beneficio tanto para el bebé como para la madre. Igualmente, se debe informar a las mujeres de la correcta aplicación de las técnicas para coadyuvar al desarrollo de los bebés desde su nacimiento en adelante; así como ir preparándolos desde que están en la etapa de gestación para generar aprendizajes que sean de provecho en la formación escolar como: las matemáticas, el lenguaje y los desafíos de la vida de los futuros niños y niñas.

En la dimensión valoración de la estimulación prenatal los médicos indican el binomio MedicoPsicopedagogo pueden orientar mejor a las mujeres gestantes en el conocimiento del estímulo prenatal, su importancia y los beneficios que presenta para sus hijos en su futuro aprendizaje. De igual manera, les gustaría fortalecer los controles prenatales con un equipo psicopedagógico para brindar sesiones de estimulación prenatal, tomando consideraciones importantes en el ámbito educativo para tener una sociedad más prospera y teniendo atenciones de mayor calidad para la mujer gestante y así poder coadyuvar en el desarrollo cognitivo y sensorial de los bebés.

Seguidamente, se procedió hacer la 
triangulación de los resultados y contrastar los resultados obtenidos y conformar un diagnóstico de la realidad estudiada. En tal sentido, se encontró que:

- Si bien existen conocimientos por parte de los tres componentes, los conocimientos que tienen son limitados; sin embargo consideran que el estímulo prenatal es de gran importancia para el desarrollo del bebé ya que aporta grandes beneficios potenciando las futuras habilidades y conocimientos. Por otra parte, los profesionales en salud desconocen las áreas y componentes de estimulación prenatal; y las mujeres gestantes indicaron que conocen del estímulo prenatal en términos generales, pero no manejan conocimientos más amplios sobre la estimulación prenatal.

- En cuanto a desarrollo sensorial, si bien los participantes conocen las técnicas de estimulación en el área sensorial desconocen su finalidad y la manera correcta de aplicarlas; así como las bases de la estimulación prenatal, la organización de cada actividad. Cuando las mujeres aplican técnicas sensoriales son por prácticas y sin la supervisión de un profesional.

- En relación a las acciones técnicas hay un desconocimiento de estas para lograr estimular el área cognitiva; las cuales ofrecen grandes beneficios, que se pierden por falta de información. Se requiere difundir la información y la aplicación correcta de cada una de las actividades, la organización y contar con la supervisión de un profesional.

- Más del noventa por ciento de las mujeres gestantes encuestadasseñalan que desean recibir más información sobre la estimulación prenatal. Por lo que es de manera urgente desarrollar un Programa de Estimulación Prenatal para un mejor desarrollo del aprendizaje temprano del bebe y una sociedad más desarrollada.

\section{DISCUSIÓN}

De acuerdo a lo estudiado en la presente investigación y la relevancia de la estimulación prenatal en el desarrollo del feto; se plantea como propuesta un programa educativo de estimulación prenatal para fortalecer el desarrollo cognitivo y sensorial del nuevo ser en mujeres gestantes atendidas en los centros de salud del municipio de potosí. Para lo cual se considera la aplicación de diferentes técnicas auditivas, visuales, táctiles, de memoria, concentración, movimiento y relajación que promuevan el desarrollo de las capacidades y habilidades del feto; permitiendo prevenir futuros problemas de aprendizaje. El objetivo del programa es: Implementar una propuesta al SEDES Potosí con programa educativo de estimulación prenatal para el desarrollo cognitivo y sensorial del nuevo ser en mujeres gestantes.

Por lo que es importante reconocer los beneficios que puede aportar dicho programa a la comunidad de Potosí, tales como:

- Es un medio favorable que ayuda a construir la inteligencia en una etapa clave

- Es importante porque le ayudara a un mejor desarrollo de su autoestima y de reconocer sus potencialidades.

- Es importante porque ayuda a desarrollar el aprendizaje infantil temprano y un mejor aprendizaje escolar a futuro.

- El programa educativo de estimulación prenatal ayuda a crear un entorno para la mujer gestante con un ambiente más tranquilo y variado eliminando el estrés cotidiano, proporcionándole bienestar físico y emocional.

La fundamentación del programa se sostiene en el ámbito teórico, psicopedagógico, sociológico y legal. Desde lo teórico a partir de las investigaciones presentadas previamente, que 
refrendan la relevancia de la estimulación prenatal en mujeres gestantes para el desarrollo del bebé y su impacto en la vida de ese nuevo ser. Desde lo psicopedagógico el programa proporciona una guía de actividades para la estimulación prenatal adecuada al contexto sociocultural de la población boliviana. La propuesta pretende desarrollar entornos educativos y saludables que sean altamente estimulantes para los futuros niños y niñas, mediante el programa educativo de estimulación prenatal que contará con componentes que dan respuestas a las necesidades de las mujeres gestantes. Desde lo sociológico el programa atiende una de las experiencias más importantes en la vida de cada mujer y su entorno familiar; pues se presentan grandes cambios físicos y emocionales. Por lo cual la madre busca ser entendida por su entorno familiar y la sociedad, para poder llevar un embarazo en armonía y felicidad. Desde lo legal la Resolución Ministerial $N^{\circ} 0420$ del 5 de Julio del 2017 indica en el artículo 12 del código de salud, que la autoridad de salud está facultada para dictar las disposiciones a las que se sujetaran los organismos públicos y privados en la elaboración y difusión de sus programas en todos los aspectos de la educación para la salud.

Para la implementación del programa de estimulación prenatal en los centros de salud se deben cumplir 3 fases en relación a los objetivos de la propuesta.

Primera Fase: Para iniciar la implementación se requiere la capacitación del personal involucrado: médicos y enfermeras que atenderán a las mujeres gestantes. Dicha capacitación tiene el objetivo formar equipos de profesionales que manejen el conocimiento base de las técnicas cognitivas y sensoriales de estimulación prenatal, necesario para llevar acabo la propuesta. Las actividades educativas serán dictadas por un psicopedagógico una vez a la semana, en sesiones de 40 minutos durante 2 meses.
Segunda Fase: En esta fase es fundamental la participación de un psicopedagógico para el diseño y ejecución las guías de técnicas cognitivas y sensoriales de estimulación. Este profesional será el encargado de implementar espacios con ambientes cómodos, agradables y apropiados para brindar sesiones de estimulación prenatal a las mujeres gestantes en los centros de salud.

Tercera Fase: En esta fase se realizarán charlas de concientización sobre la importancia de la estimulación prenatal a mujeres que se encuentren entre el segundo y tercer trimestre de gestación que no presenten ninguna patología. Las charlas serán de 15 a 20 minutos, 1 veces por semana durante 1 mes. Las mujeres interesadas se les difundirá técnicas cognitivas y sensoriales de estimulación prenatal por 30 a 40 minutos en 1 sesión por semana durante 3 meses, las sesiones serán de manera dinámica y participativa.

\section{CONCLUSIONES}

Se ha determinado que existe una relación teórica y metodológica en el Proceso de Estimulación Prenatal percatándose de las características conceptuales que tiene en el área de Salud con la intervención del psicopedagogo.

El Servicio Departamental de Salud no oferta servicios de Estimulación prenatal como Programa por ello existe un desconocimiento por parte de los profesionales y de las mujeres gestantes en lo que refiere la importancia, los beneficios, como las técnicas para el buen desarrollo de un nuevo ser.

Existe la necesidad de implementar el servicio de Estimulación Prenatal como parte de los servicios de salud integral siendo que las mujeres gestantes señalan que les gustaría tener un Programa de Estimulación Prenatal en sus diferentes Centros de Salud. 


\section{REFERENCIAS}

Bertin, M. (2018). La Educación Prenatal Natural. Madrid: Panamericana.

Corominas, C. (2011). Embarazo y Primer Año de Vida. En C. Corominas, Embarazo y Primer Año de Vida (pág. 288). España: Palabra.

García García, L. (2008). Prácticas sobre estimulación prenatal que realizan las gestantes adultas asistentes al control prenatal en Sincelejo (Colombia). Salud Uninorte, 24, (1): 31-39. http://www.scielo.org.co/pdf/sun/v24n1/ v24n1a05.pdf

García Montaner, N. (2019). Definición de embarazo y sus fases. https://www.salud.mapfre.es/saludfamiliar/mujer/embarazo/definicion/

González, G. (2016). ¿Cómo afrontar el embarazo adolescente?. Facemama. www.facemama.com

Gútiez, (2016). Neurociencia y Educación Prenatal. Boletín del Colegio Oficial de Doctores y Licenciados en Filosofía y Letras y en Ciencias. (260). https://www.cdlmadrid.org/archivos/ boletin_marzo_2016.pdf
Guzmán, L. (2014). Estimulación en la etapa prenatal. Tesis de Grado. México: Universidad Rafael Landivar. http://biblio3.url.edu.gt/ Tesario/2014/05/84/Guzman-Lesly.pdf

Kinedu. (2017). La historia de la estimulación prenatal. https://blog-es.kinedu.com/lahistoria-de-la-estimulacion-prenatal/

López Fuentes, B. (2016). Cuidados en el Embarazo. Buenos Aires.

Piaget, J. (1969) Psicología y Pedagogía. Barcelona: Ariel.

Rodríguez, R. (2018). Alimentación para Mujeres Gestantes. Colombia: Océano Medicina,

Romero, M. I. (2007). Embarazo en la adolescencia. http://escuela.med.puc.cl/paginas/ops/curso/ lecciones/leccion15/M3L15Bibliografia.html Verny, T. (1988). La vida secreta del niño antes de nacer. Argentina: Editorial Urano 